\title{
CONVERGENCE AND APPROXIMATION OF THE STURM-LIOUVILLE OPERATORS WITH POTENTIALS-DISTRIBUTIONS
}

\section{A. S. Horyunov}

UDC 517.984.5

\begin{abstract}
We study the operators $L_{n} y=-\left(p_{n} y^{\prime}\right)^{\prime}+q_{n} y, n \in \mathbb{Z}_{+}$, given on a finite interval with various boundary conditions. It is assumed that the function $q_{n}$ is a derivative (in a sense of distributions) of $Q_{n}$ and $1 / p_{n}$, $Q_{n} / p_{n}$, and $Q_{n}^{2} / p_{n}$ are integrable complex-valued functions. The sufficient conditions for the uniform convergence of Green functions $G_{n}$ of the operators $L_{n}$ on the square as $n \rightarrow \infty$ to $G_{0}$ are established. It is proved that every $G_{0}$ is the limit of Green functions of the operators $L_{n}$ with smooth coefficients. If $p_{0}>0$ and $Q_{0}(t) \in \mathbb{R}$, then they can be chosen so that $p_{n}>0$ and $q_{n}$ are real-valued and have compact supports.
\end{abstract}

\section{Introduction}

The theory of Sturm-Liouville operators is one of the most developed fields in the theory of ordinary differential equations (see, e.g., the monograph [1] and the references therein).

The main object of this theory is the expression

$$
l(y)=-\left(p(t) y^{\prime}(t)\right)^{\prime}+q(t) y(t)
$$

defined on a finite segment $[a, b]$ and the operators connected with this expression. Usually, it is assumed that the coefficients of (1) satisfy the following conditions:

$$
1 / p, \quad q \in L_{1}([a, b] ; \mathbb{C})
$$

Physicists are interested in the case where the function $q$ in the differential expression (1) is a measure or even a more singular generalized function (see, e.g., the monographs $[2,3]$ and the references therein).

An approach proposed in $[4,5]$ (see also [6]) enables one to give a correct definition of the differential expression (1) under much more general conditions imposed on the coefficients

$$
q=Q^{\prime}, \quad 1 / p, \quad Q / p, \quad Q^{2} / p \in L_{1}([a, b] ; \mathbb{C}),
$$

where the derivative $Q^{\prime}$ is understood in a sense of generalized functions. This approach is based on the theory of quasidifferential Shin-Zettl operators $[7,8]$ and enables one to investigate differential operators of higher orders [5,9]. A natural question arises concerning the possibility of representation of the differential operator generated by expression (1) and homogeneous two-point boundary conditions in the form of the uniform resolvent limit (see [10]) of similar operators with smooth coefficients. For $p(t) \equiv 1$, the affirmative answer to this question was given in $[11,12]$. The case where $p(t)>0$ almost everywhere on $[a, b]$ and the function $Q$ is real-valued was studied in [13]. In the present paper, we generalize and strengthen this result and formulate it via the uniform approximation of the Green function.

Institute of Mathematics, Ukrainian National Academy of Sciences, Kyiv, Ukraine.

Translated from Ukrains'kyi Matematychnyi Zhurnal, Vol. 67, No. 5, pp. 602-610, May, 2015. Original article submitted March 23, 2015. 


\section{Preliminary Results}

We first present necessary results from [4]. For a function given on the segment $[a, b]$, we introduce the quasiderivatives

$$
\begin{aligned}
& D^{[0]} y=y, \\
& D^{[1]} y=p y^{\prime}-Q y, \\
& D^{[2]} y=\left(D^{[1]} y\right)^{\prime}+\frac{Q}{p} D^{[1]} y+\frac{Q^{2}}{p} y .
\end{aligned}
$$

Denote

$$
\widehat{y}(t)=\left(D^{[0]} y(t), D^{[1]} y(t)\right) \in \mathbb{C}^{2} .
$$

Under assumptions (2), the quantities $D^{[0]} y(t), D^{[1]} y(t)$, and $D^{[2]} y(t)$ are Shin-Zettl quasiderivatives (see [8], Chap. 1). One can also easily show that, for sufficiently smooth functions $p$ and $Q$ (corresponding to the classical Sturm-Liouville expression), the following equality is true:

$$
l(y)=-D^{[2]} y .
$$

Hence, the formal expression (1) can be correctly defined as the quasidifferential Shin-Zettl expression

$$
l[y]=-D^{[2]} y .
$$

The corresponding Shin-Zettl matrix has the form

$$
A(t)=\left(\begin{array}{cc}
\frac{Q}{p} & \frac{1}{p} \\
-\frac{Q^{2}}{p} & -\frac{Q}{p}
\end{array}\right) \in L_{1}\left([a, b] ; \mathbb{C}^{2 \times 2}\right) .
$$

Consider a two-point quasidifferential boundary-value problem

$$
\begin{gathered}
l[y]=f(t) \in L_{1}([a, b], \mathbb{C}), \\
\alpha \widehat{y}(a)+\beta \widehat{y}(b)=0,
\end{gathered}
$$

where the matrices $\alpha, \beta \in \mathbb{C}^{m \times m}$.

The following statement connects the quasidifferential boundary-value problem (4), (5) with systems of firstorder differential equations:

Lemma 1. A function $y(t)$ is a solution of the boundary-value problem (4), (5) if and only if the vector function $w(t)=\widehat{y}(t)$ is a solution of the boundary-value problem

$$
\begin{gathered}
w^{\prime}(t)=A(t) w(t)+\varphi(t), \\
\alpha w(a)+\beta w(b)=0,
\end{gathered}
$$

where the square-matrix function $A(t)$ is given by relation (3) and $\varphi(t)=(0,-f(t)) \in L_{1}\left([a, b] ; \mathbb{C}^{2}\right)$. 
Assume that the homogeneous boundary-value problem

$$
w^{\prime}(t)=A(t) w(t), \quad \alpha w(a)+\beta w(b)=0
$$

possesses only a trivial solution. It is known that, in this case, there exists a Green function of this problem

$$
G(t, s)=\left(\begin{array}{ll}
g_{11}(t, s) & g_{12}(t, s) \\
g_{21}(t, s) & g_{22}(t, s)
\end{array}\right) \in L_{\infty}\left([a, b] ; \mathbb{C}^{2 \times 2}\right)
$$

which has the form

$$
G(t, s)= \begin{cases}-Y(t)(\alpha+\beta Y(b))^{-1} \beta Y(b) Y^{-1}(s), & a \leq t<s \\ Y(t)\left[I_{2}-(\alpha+\beta Y(b))^{-1} \beta Y(b)\right] Y^{-1}(s), & s<t \leq b\end{cases}
$$

where $I_{2}$ is the $(2 \times 2)$ identity matrix and $Y(t)$ is a matricant, i.e., the solution of the matrix Cauchy problem

$$
Y^{\prime}(t)=A(t) Y(t), \quad Y(a)=I_{2}
$$

A unique solution of the boundary-value problem (6), (7) can be expressed via the Green matrix as follows:

$$
w(t)=\int_{a}^{b} G(t, s) \varphi(s) d s, \quad t \in[a, b] .
$$

We introduce a similar object for the quasidifferential boundary-value problem (4), (5).

Definition 1. A Green function of the semihomogeneous boundary-value problem (4), (5) is defined as a continuous function $\Gamma(t, s) \in C([a, b] \times[a, b], \mathbb{C})$ with the help of which the solution of the indicated problem can be represented in the form

$$
y(t)=\int_{a}^{b} \Gamma(t, s) f(s) d s
$$

Theorem 1. Assume that the homogeneous boundary-value problem

$$
D^{[2]} y(t)=0, \quad \alpha \widehat{y}(a)+\beta \widehat{y}(b)=0
$$

has only the trivial solution.

Then there exists a unique Green function $\Gamma(t, s)$ of the boundary-value problem (4), (5) and

$$
\Gamma(t, s)=-g_{12}(t, s)
$$

Proof. By Lemma 1, the assumption of the theorem implies that the homogeneous boundary-value problem

$$
w^{\prime}(t)=A(t) w(t), \quad \alpha w(a)+\beta w(b)=0
$$


also has solely the trivial solution and, hence, the Green function $G(t, s)$ exists for problem (6), (7) and equality (9) is true.

We apply Lemma 1 once again and rewrite relation (9) in the form

$$
\begin{aligned}
& D^{[0]} y(t)=-\int_{a}^{b} g_{12}(t, s) f(s) d s, \\
& D^{[1]} y(t)=-\int_{a}^{b} g_{22}(t, s) f(s) d s,
\end{aligned}
$$

where $y(t)$ is a unique solution of problem (4), (5).

According to relation (8), all off-diagonal elements of the matrix $G(t, s)$ are continuous functions in view of the continuity of the matricant $Y(t)$ and $Y^{-1}(t)$.

This yields the existence of the Green function.

Now let $\Gamma^{\prime}(t, s)$ be a different Green function of the boundary-value problem (4), (5).

Then, for any function $f \in L_{1}([a, b], \mathbb{C})$, we can represent the unique solution of this problem in the form

$$
y(t)=\int_{a}^{b} \Gamma(t, s) f(s) d s=\int_{a}^{b} \Gamma^{\prime}(t, s) f(s) d s,
$$

i.e.,

$$
\int_{a}^{b}\left(\Gamma^{\prime}(t, s)-\Gamma(t, s)\right) f(s) d s=0 .
$$

Hence, the bounded kernel $\Gamma^{\prime}(t, s)-\Gamma(t, s)$ generates a zero integral operator.

In this case, it is known that $\Gamma^{\prime}(t, s)-\Gamma(t, s)=0$ almost everywhere on $[a, b]$. In view of the continuity of the functions $\Gamma(t, s)$ and $\Gamma^{\prime}(t, s)$, this yields the uniqueness of the Green function.

Theorem 1 is proved.

\section{Convergence of Green Functions}

Parallel with $l(y)$, we consider a family of Sturm-Liouville expressions $l_{n}(y)$ of the form (1) with coefficients

$$
p_{n}, q_{n}=Q_{n}^{\prime}, \quad n \in \mathbb{N},
$$

satisfying conditions (2). We denote the quasiderivatives corresponding to these expressions by $D_{n}^{[0]} y, D_{n}^{[1]} y$, and $D_{n}^{[2]} y$ and the corresponding vector of quasiderivatives by

$$
\widehat{y}_{n}(t):=\left(D_{n}^{[0]} y(t), D_{n}^{[1]} y(t)\right) \in \mathbb{C}^{2}
$$

the corresponding Shin-Zettl matrices are denoted by $A_{n}(t)$ and the quasidifferential expressions by $l_{n}[y]$.

Parallel with problem (4), (5), for each $n$, we consider the following boundary-value problems:

$$
\begin{gathered}
l_{n}[y](t)=f_{n}(t) \in L_{2}([a, b] ; \mathbb{C}), \\
\alpha_{n} \widehat{y}_{n}(a)+\beta_{n} \widehat{y}_{n}(b)=0 .
\end{gathered}
$$


By Lemma 1, they are equivalent to the boundary-value problems

$$
\begin{gathered}
w^{\prime}(t)=A_{n}(t) w(t)+\varphi_{n}(t), \\
\alpha_{n} w(a)+\beta_{n} w(b)=0,
\end{gathered}
$$

where $w(t)=\widehat{y}_{n}(t)$ and $\varphi_{n}(t)=\left(0,-f_{n}(t)\right) \in L_{1}\left([a, b] ; \mathbb{C}^{2}\right)$.

Theorem 2. Let the following conditions be satisfied:

(i) the homogeneous boundary-value problem

$$
D^{[2]} y(t)=0, \quad \alpha \widehat{y}(a)+\beta \widehat{y}(b)=0
$$

possesses solely the trivial solution;

(ii) the coefficients of expressions satisfy the following limit relations as $n \rightarrow \infty$ :

(a) $\left\|1 / p_{n}\right\|_{1}=O(1),\left\|Q_{n} / p_{n}\right\|_{1}=O(1)$, and $\left\|Q_{n}^{2} / p_{n}\right\|_{1}=O(1)$

(b) $\left\|\int_{a}^{t}\left(1 / p_{n}-1 / p\right) d s\right\|_{\infty} \rightarrow 0$

(c) $\left\|\int_{a}^{t}\left(Q_{n} / p_{n}-Q / p\right) d s\right\|_{\infty} \rightarrow 0$

(d) $\left\|\int_{a}^{t}\left(Q_{n}^{2} / p_{n}-Q^{2} / p\right) d s\right\|_{\infty} \rightarrow 0$

(iii) the matrices specifying the boundary conditions satisfy the limit relations $\alpha_{n} \rightarrow \alpha$ and $\beta_{n} \rightarrow \beta$ as $n \rightarrow \infty$.

Then, for sufficiently large $n$, there exist Green functions $\Gamma_{n}(t, s)$ of the semihomogeneous boundary-value problems (10), (11) and the limit relation

$$
\left\|\Gamma_{n}(t, s)-\Gamma(t, s)\right\|_{\infty} \rightarrow 0, \quad n \rightarrow \infty
$$

is true.

Here and in what follows, $\|\cdot\|_{\infty}$ is the sup-norm and $\|\cdot\|_{p}$ is the norm in the Lebesgue space $L_{p}, p \geq 1$.

Remark 1. It is obvious that conditions (ii) are satisfied if, as $n \rightarrow \infty$,

$$
\left\|1 / p_{n}-1 / p\right\|_{1} \rightarrow 0, \quad\left\|Q_{n} / p_{n}-Q / p\right\|_{1} \rightarrow 0, \quad\left\|Q_{n}^{2} / p_{n}-Q^{2} / p\right\|_{1} \rightarrow 0
$$

The proof of the theorem is based on the auxiliary result from [15]. Note that this result was later generalized in [14] (see also the references therein).

By $Y_{n}(\cdot)$ we denote the matricants corresponding to problems (12), (13), i.e., the solutions of the matrix Cauchy problems

$$
Y_{n}^{\prime}(t)=A_{n}(t) Y_{n}(t), \quad Y_{n}(a)=\left(\begin{array}{ll}
1 & 0 \\
0 & 1
\end{array}\right)
$$


Lemma 2. If one of the following four (nonequivalent) conditions is satisfied as $n \rightarrow \infty$ :

( $\alpha) \quad\left\|A_{n}-A\right\|_{1}=O(1)$,

( $\beta)\left\|\int_{a}^{t}\left(A_{n}(s)-A(s)\right) d s \cdot\left(A_{n}(t)-A(t)\right)\right\|_{1} \rightarrow 0$,

$(\gamma)\left\|\left(A_{n}(t)-A(t)\right) \cdot \int_{a}^{t}\left(A_{n}(s)-A(s)\right) d s\right\|_{1} \rightarrow 0$,

( $\delta)\left\|\int_{a}^{t}\left(A_{n}(s)-A(s)\right) d s\left(A_{n}(t)-A(t)\right)-\left(A_{n}(t)-A(t)\right) \int_{a}^{t}\left(A_{n}(s)-A(s)\right) d s\right\|_{1} \rightarrow 0$,

then the condition

$$
\left\|\int_{a}^{t}\left(A_{n}(s)-A(s)\right) d s\right\|_{\infty} \rightarrow 0
$$

is equivalent to the convergence

$$
\left\|Y_{n}-Y\right\|_{\infty} \rightarrow 0, \quad\left\|Y_{n}^{-1}-Y^{-1}\right\|_{\infty} \rightarrow 0
$$

as $n \rightarrow \infty$.

Proof of Theorem 2. By Lemma 1, it follows from the assumption (i) of Theorem 2 that the homogeneous boundary-value problems

$$
w^{\prime}(t)=A_{n}(t) w(t), \quad \alpha_{n} w(a)+\beta_{n} w(b)=0
$$

also have only trivial solutions for sufficiently large $n$. By Theorem 1, the Green functions of problems (10), (11) exist.

We now prove relation (14).

It is easy to see that condition (i) of Theorem 2 implies condition $(\alpha)$ of Lemma 2 and condition (ii) yields the condition

$$
\left\|\int_{a}^{t}\left(A_{n}(s)-A(s)\right) d s\right\|_{\infty} \rightarrow 0 .
$$

Hence, by Lemma 2, the limit relation (15) is true. In view of relation (8), this implies that the limit equality (14) holds.

Theorem 2 is proved.

\section{Approximation of Green Functions}

We now proceed to the problem of approximation. We again consider an expression $l(y)$ of the form (1) whose coefficients satisfy conditions (ii) and the boundary-value problem (4), (5) generated by this expression.

Theorem 3. Let the conditions of Theorem 1 be satisfied. Then there exist $p_{n}, Q_{n}, n \in \mathbb{N}$, such that $p_{n} \in C^{\infty}([a, b], \mathbb{C}), Q_{n} \in C_{0}^{\infty}([a, b], \mathbb{C})$, and condition (ii) of Theorem 2 is satisfied, i.e., for problem (4), (5), 
one can construct a sequence of Sturm-Liouville problems with smooth coefficients $p_{n}$ and $q_{n}$ such that the limit relation (14) is true.

Furthermore, if the functions $p$ and $Q$ are real-valued and $p>0$ almost everywhere on $[a, b]$, then the smooth functions $p_{n}$ and $Q_{n}$ (and, hence, $q_{n}$ ) can be chosen in the same way.

Proof. Since $\frac{1}{p} \in L_{1}([a, b], \mathbb{C})$, we conclude that $p(t) \neq 0$ almost everywhere on $[a, b]$. By $\tilde{p}_{n}$ we denote the Sobolev average of the function $\frac{1}{\sqrt{p}} \in L_{2}([a, b], \mathbb{C})$ and define $p_{n}:=\frac{1}{\tilde{p}_{n}^{2}}$.

Then

$$
p_{n} \in C^{\infty}([a, b], \mathbb{C}), \quad\left\|\frac{1}{\sqrt{p_{n}}}-\frac{1}{\sqrt{p}}\right\|_{2} \rightarrow 0, \quad n \rightarrow \infty
$$

The condition of the theorem also implies that $\frac{Q}{\sqrt{p}} \in L_{2}([a, b], \mathbb{C})$. Since the set $C_{0}^{\infty}([a, b], \mathbb{C})$ is dense in the space $L_{2}([a, b], \mathbb{C})$, we can choose $\widetilde{Q}_{n} \in C_{0}^{\infty}([a, b], \mathbb{C})$ such that

$$
\left\|\widetilde{Q}_{n}-\frac{Q}{\sqrt{p}}\right\|_{2} \rightarrow 0 \quad n \rightarrow \infty
$$

Setting $Q_{n}:=\widetilde{Q}_{n} \sqrt{p_{n}}$, we get

$$
Q_{n} \in C_{0}^{\infty}([a, b], \mathbb{C}), \quad\left\|\frac{Q_{n}}{\sqrt{p_{n}}}-\frac{Q}{\sqrt{p}}\right\|_{2} \rightarrow 0, \quad n \rightarrow \infty .
$$

Further, we obtain

$$
\begin{aligned}
\left\|\frac{Q_{n}}{p_{n}}-\frac{Q}{p}\right\|_{1} & =\left\|\frac{Q_{n}}{p_{n}}-\frac{Q}{\sqrt{p_{n}} \sqrt{p}}+\frac{Q}{\sqrt{p_{n}} \sqrt{p}}-\frac{Q}{p}\right\|_{1} \\
\leq & \left\|\frac{1}{\sqrt{p_{n}}}\right\|_{2}\left\|\frac{Q_{n}}{\sqrt{p_{n}}}-\frac{Q}{\sqrt{p}}\right\|_{2}+\left\|\frac{Q}{\sqrt{p}}\right\|_{2}\left\|\frac{1}{\sqrt{p_{n}}}-\frac{1}{\sqrt{p}}\right\|_{2}, \\
\left\|\frac{1}{p_{n}}-\frac{1}{p}\right\|_{1} & =\left\|\left(\frac{1}{\sqrt{p_{n}}}-\frac{1}{\sqrt{p}}\right)\left(\frac{1}{\sqrt{p_{n}}}+\frac{1}{\sqrt{p}}\right)\right\|_{1} \\
\leq & \left\|\frac{1}{\sqrt{p_{n}}}-\frac{1}{\sqrt{p}}\right\|_{2}\left\|\frac{1}{\sqrt{p_{n}}}+\frac{1}{\sqrt{p}}\right\|_{2}, \\
\left\|\frac{Q_{n}^{2}}{p_{n}}-\frac{Q^{2}}{p}\right\|_{1} & =\left\|\left(\frac{Q_{n}}{\sqrt{p_{n}}}-\frac{Q}{\sqrt{p}}\right)\left(\frac{Q_{n}}{\sqrt{p_{n}}}+\frac{Q}{\sqrt{p}}\right)\right\|_{1} \\
\leq & \leq \frac{Q_{n}}{\sqrt{p_{n}}}-\frac{Q}{\sqrt{p}}\left\|_{2}\right\| \frac{Q_{n}}{\sqrt{p_{n}}}+\frac{Q}{\sqrt{p}} \|_{2},
\end{aligned}
$$

and, hence, the conditions of Remark 1 are satisfied.

Theorem 3 is proved. 


\section{Convergence and Approximation of Operators}

Here, we also present some necessary results from [4].

In the Hilbert space $L_{2}([a, b] ; \mathbb{C})$ (see $[7,8]$ ), a quasidifferential expression $l[y]$ generates a maximal quasidifferential operator

$$
\begin{gathered}
L_{\max }: y \rightarrow l[y] \\
\operatorname{Dom}\left(L_{\max }\right)=\left\{y \mid D^{[k]} y \in A C([a, b] ; \mathbb{C}), k=\overline{0, m-1}, D^{[m]} y \in L_{2}([a, b] ; \mathbb{C})\right\} .
\end{gathered}
$$

A minimal quasidifferential operator is defined as the restriction of the operator $L_{\max }$ to a linear manifold

$$
\operatorname{Dom}\left(L_{\text {min }}\right):=\left\{y \in \operatorname{Dom}\left(L_{\text {max }}\right) \mid \widehat{y}(a)=\widehat{y}(b)=0\right\}
$$

Remark 2. It is clear that the quasiderivatives $D^{[1]} y$ and $D^{[2]} y$ depend on the choice of the primitive $Q$ (to within a constant). It is easy to see that the operators $L_{\min }$ and $L_{\max }$ remain unchanged.

Parallel with (1), we consider the formally adjoint differential expression

$$
l^{+}(y)=\left(-\bar{p}(t) y^{\prime}(t)\right)^{\prime}+\bar{q}(t) y(t)
$$

where the bar stands for the complex conjugation. By $L_{\max }^{+}$and $L_{\min }^{+}$we denote, respectively, the maximum and minimum quasidifferential operators in the space $L_{2}([a, b] ; \mathbb{C})$. By using the results presented in [8] for the general quasidifferential Shin-Zettl expressions and the facts established above, we conclude that the operators $L_{\min }, L_{\min }^{+}, L_{\max }$, and $L_{\max }^{+}$are densely defined and closed in the space $L_{2}([a, b] ; \mathbb{C})$,

$$
L_{\min }^{*}=L_{\max }^{+}, \quad \text { and } \quad L_{\max }^{*}=L_{\min }^{+}
$$

Similarly, for each $n$, the expressions $l_{n}[y]$ generate the operators $L_{\min }^{n}$ and $L_{\max }^{n}$ in the Hilbert space $L_{2}([a, b] ; \mathbb{C})$.

In [5], one can find the description of some classes of extensions of the minimal quasidifferential operator $L_{\text {min }}$ under condition of its symmetry. Here, we consider an arbitrary extension of a minimal (generally speaking, nonsymmetric) operator specified by the two-point boundary conditions. Namely, we consider the operator

$$
L y=l[y]
$$

$$
\operatorname{Dom}(L)=\left\{y \in \operatorname{Dom}\left(L_{\max }\right) \mid \alpha \widehat{y}(a)+\beta \widehat{y}(b)=0\right\},
$$

corresponding to problem (4), (5) and the operators

$$
\begin{gathered}
L_{n} y=l_{n}[y], \\
\operatorname{Dom}\left(L_{n}\right)=\left\{y \in \operatorname{Dom}\left(L_{\max }^{n}\right) \mid \alpha_{n} \widehat{y}_{n}(a)+\beta_{n} \widehat{y}_{n}(b)=0\right\},
\end{gathered}
$$

corresponding to boundary-value problems (10), (11).

It is clear that $L_{\min } \subset L \subset L_{\max }$ and $L_{\text {min }}^{n} \subset L_{n} \subset L_{\text {max }}^{n}$. 
Theorem 4. Assume that the resolvent set of a boundary operator $\rho(L)$ is nonempty and that conditions (ii) and (iii) of Theorem 2 are satisfied as $n \rightarrow \infty$.

Then, for any $\lambda \in \rho(L), \lambda \in \rho\left(L_{n}\right)$ for sufficiently large $n$ and

$$
\left\|\left(L_{n}-\lambda\right)^{-1}-(L-\lambda)^{-1}\right\|_{H S} \rightarrow 0, \quad n \rightarrow \infty,
$$

where $\|\cdot\|_{H S}$ is the Hilbert-Schmidt norm.

Proof. We first assume that $0 \in \rho(L)$. This implies that the operator $L$ is invertible, i.e., the problem $L y=f$ is equivalent to problem (4), (5) and, for any $f \in L_{2}([a, b], \mathbb{C})$, has a unique solution, which is equivalent to condition (i) of Theorem 2.

This solution $y(t)$ can be represented in the form

$$
y(t)=\int_{a}^{b} \Gamma(t, s) f(s) d s .
$$

By Theorem 2, the operators $L_{n}$ are also invertible, there exist Green functions of the corresponding boundaryvalue problems (10), (11), and their solutions have the form

$$
y_{n}(t)=\int_{a}^{b} \Gamma_{n}(t, s) f(s) d s .
$$

Hence,

$$
\begin{aligned}
\left\|L_{n}^{-1}-L^{-1}\right\|_{H S} & =\left(\int_{a}^{b} \int_{a}^{b}\left|\Gamma_{n}(t, s)-\Gamma(t, s)\right|^{2} d t d s\right)^{1 / 2} \\
& \leq\left\|\Gamma_{n}(t, s)-\Gamma(t, s)\right\|_{\infty} \cdot(b-a) \rightarrow 0, \quad n \rightarrow \infty .
\end{aligned}
$$

We now consider the general case. Thus, there exists some $\lambda \in \rho(L)$. Then it is clear that $0 \in \rho(L-\lambda)$.

We consider the operator $L-\lambda$. The problem $(L-\lambda) y=f$ is equivalent to the boundary-value problem

$$
\begin{gathered}
l[y]-\lambda y=f(t) \in L_{1}([a, b], \mathbb{C}), \\
\alpha \widehat{y}(a)+\beta \widehat{y}(b)=0 .
\end{gathered}
$$

Note that Lemma 1 with the matrix $A=A_{\lambda}$ is true for this problem. It is easy to see that the matrices $A$ and $A_{n}$, together with the matrices $A_{\lambda}$ and $A_{n \lambda}$, satisfy the conditions of Theorem 2.

Repeating the reasoning presented above, we conclude that $0 \in \rho\left(L_{n}-\lambda\right)$ for sufficiently large $n$, there exist Green functions of the corresponding boundary-value problems, and the limit relation (16) is true.

Remark 3. Theorem 4 yields the uniform resolvent convergence of the operators $L_{n}$ to $L$, which was established in [4].

Remark 4. By analogy with Remark 1, the following conditions for the coefficients of the expression as $n \rightarrow \infty$ are sufficient for the convergence of resolvents of operators (16):

$$
\left\|1 / p_{n}-1 / p\right\|_{1} \rightarrow 0, \quad\left\|Q_{n} / p_{n}-Q / p\right\|_{1} \rightarrow 0, \quad\left\|Q_{n}^{2} / p_{n}-Q^{2} / p\right\|_{1} \rightarrow 0
$$


Theorems 3 and 4 lead to the following result:

Theorem 5. Assume that the quasidifferential operator L corresponding to the formal Sturm-Liouville expression $l(y)$ and satisfying conditions (2) has a nonempty resolvent set $\rho(L)$.

Then there exists a sequence of classical Sturm-Liouville operators with smooth coefficients such that their resolvents approximate the resolvent of the operator $L$ in the Hilbert-Schmidt norm, i.e., relation (16) is true.

\section{REFERENCES}

1. A. Zettl, Sturm-Liouville Theory, American Mathematical Society, Providence (2005).

2. S. Albeverio, F. Gestezy, R. Hoegh-Krohn, and H. Holden, Solvable Models in Quantum Mechanics, Springer, New York (1988).

3. S. Albeverio and P. Kurasov, Singular Perturbations of Differential Operators, Cambridge Univ. Press, Cambridge (2000).

4. A. S. Goriunov and V. A. Mikhailets, "Regularization of singular Sturm-Liouville equations," Meth. Funct. Anal. Topol., 16, No. 2, 120-130 (2010).

5. A. S. Goriunov, V. A. Mikhailets, and K. Pankrashkin, "Formally self-adjoint quasi-differential operators and boundary-value problems," Electron. J. Different. Equat., No. 101, 1-16 (2013).

6. J. Eckhardt, F. Gesztesy, R. Nichols, and G. Teschl, "Weyl-Titchmarsh theory for Sturm-Liouville operators with distributional coefficients," Opusc. Math., 33, No. 3, 467-563 (2013).

7. A. Zettl, "Formally self-adjoint quasi-differential operators," Rocky Mountain J. Math., 5, No. 3, 453-474 (1975).

8. W. N. Everitt and L. Markus, Boundary-Value Problems and Symplectic Algebra for Ordinary Differential and Quasi-Differential Operators, American Mathematical Society, Providence, RI (1999).

9. A. S. Goriunov and V. A. Mikhailets, "Regularization of two-term differential equations with singular coefficients by quasiderivatives," Ukr. Math. J., 63, No. 9, 1190-1205 (2011).

10. T. Kato, Perturbation Theory for Linear Operators, Springer, Berlin (1995).

11. A. Savchuk and A. Shkalikov, "Sturm-Liouville operators with singular potentials," Math. Notes, 66, No. 5-6, 741-753 (1999).

12. A. S. Goriunov and V. A. Mikhailets, "Resolvent convergence of Sturm-Liouville operators with singular potentials," Math. Notes, 87, No. 1-2, 287-292 (2010).

13. J. Yan and G. Shi, "Inequalities among eigenvalues of Sturm-Liouville problems with distribution potentials," J. Math. Anal. Appl., 409, No. 1, 509-520 (2014).

14. T. I. Kodlyuk, V. A. Mikhailets, and N. V. Reva, "Limit theorems for one-dimensional boundary-value problems," Ukr. Math. J., 65, No. 1, 77-90 (2013).

15. A. Yu. Levin, "Limit Transition for the nonsingular systems $\dot{X}=A_{n}(t) X$, Dokl. Akad. Nauk SSSR, 176, No. 4, 774-777 (1967). 